\title{
Public health challenges and legacies of Japan's response to the Ebola virus disease outbreak in West Africa 2014 to 2015
}

T Saito ${ }^{1}$

1. Department of Health Crisis Management, National Institute of Public Health, Saitama, Japan

Correspondence: Tomoya Saito (tsaito@niph.go.jp)

Citation style for this article:

Saito T. Public health challenges and legacies of Japan's response to the Ebola virus disease outbreak in West Africa 2014 to 2015 . Euro Surveill.

2015;20(44): pii=30056. DOI: http://dx.doi.org/10.2807/1560-7917.ES.2015.20.44.30056

Article submitted on 13 May 2015 / accepted on 28 October 2015 / published on 05 November 2015

The largest outbreak of Ebola virus disease occurred in West Africa in 2014 and resulted in unprecedented transmission even in distant countries. In Japan, only nine individuals were screened for Ebola and there was no confirmed case. However, the government promoted the reinforcement of response measures and interagency collaboration, with training and simulation exercises conducted country-wide. The legacies included: publication of a communication policy on case disclosure, a protocol for collaboration between public health and other agencies, and establishing an expert committee to assemble the limited available expertise. There were challenges in taking proportionate and flexible measures in the management of people identified to be at high risk at entry points to Japan, in the decentralised medical response strategy, and in the medical countermeasures preparedness. The Ebola outbreak in West Africa provided a crucial opportunity to reveal the challenges and improve the preparedness for rare but high impact emerging diseases that are prone to be neglected. Efforts to uphold the lessons learnt and maintain public health preparedness should help prepare for future emerging diseases, including bioterrorist acts and pandemics.

\section{Introduction}

The outbreak of Ebola virus disease (EVD) in West Africa in 2014 was the largest outbreak in history, and the World Health Organization (WHO) declared a Public Health Emergency of International Concern (PHEIC) on 8 August 2014 [1]. Importation and transmission to non-endemic countries and evacuation and repatriation outside Africa [2,3] forced public health authorities to prepare for EVD even in countries far away from the outbreak.

Japan has not experienced a case of viral haemorrhagic fever (VHF) since 1987, when a case of Lassa fever was imported from Sierra Leone [4]. The Act on Prevention of Infectious Diseases and Medical Care for Patients with Infections in Japan (the Act), which came into effect in 1999, categorises EVD as a Category 1 infectious disease (Category 1 disease), along with other viral haemorrhagic fevers, plague, and smallpox [5]. The Ministry of Health, Labour and Welfare (MHLW) and the local prefectures have designated specified infectious disease hospitals (Specified hospitals) and Class 1 infectious disease hospitals (Class 1 hospitals), respectively, to provide treatment for Category 1 diseases in an isolated biosafety ward at public expense (Table 1). Category 1 diseases are also quarantine diseases in the Quarantine Act.

Although the number of travellers with citizenship of the three EVD endemic countries was limited to approximately 300 to 500 per year [6] and no direct flight is operated to and from those countries, the Government of Japan (GOJ) started to reinforce the border controls and domestic response capacity from August 2014 onwards. As of the end of September 2015, at least 20 healthcare workers from Japan have been deployed through the WHO Global Outbreak Alert and Response Network (GOARN) network. Only nine people were screened for EVD in Japan up to September 2015 (Table 2) [7]. All were negative and no case was reported among Japanese citizens overseas either. Still a government-wide response system promoted reinforcement of response measures and interagency collaboration.

Here, the author reviews the public health and medical preparedness and response in Japan to the 2014 EVD outbreak in West Africa, and discusses the legacies and challenges for the preparedness and response to emerging diseases.

\section{Border measures and management of people travelling to Japan from endemic countries}

The GOJ reinforced border measures for containment of EVD from August 2014 by raising awareness among 
Case management protocol for people with a history of travel to Ebola virus disease endemic countries within the previous 21 days, Japan, 21 November 2014-17 September 2015 [modified from reference 9]

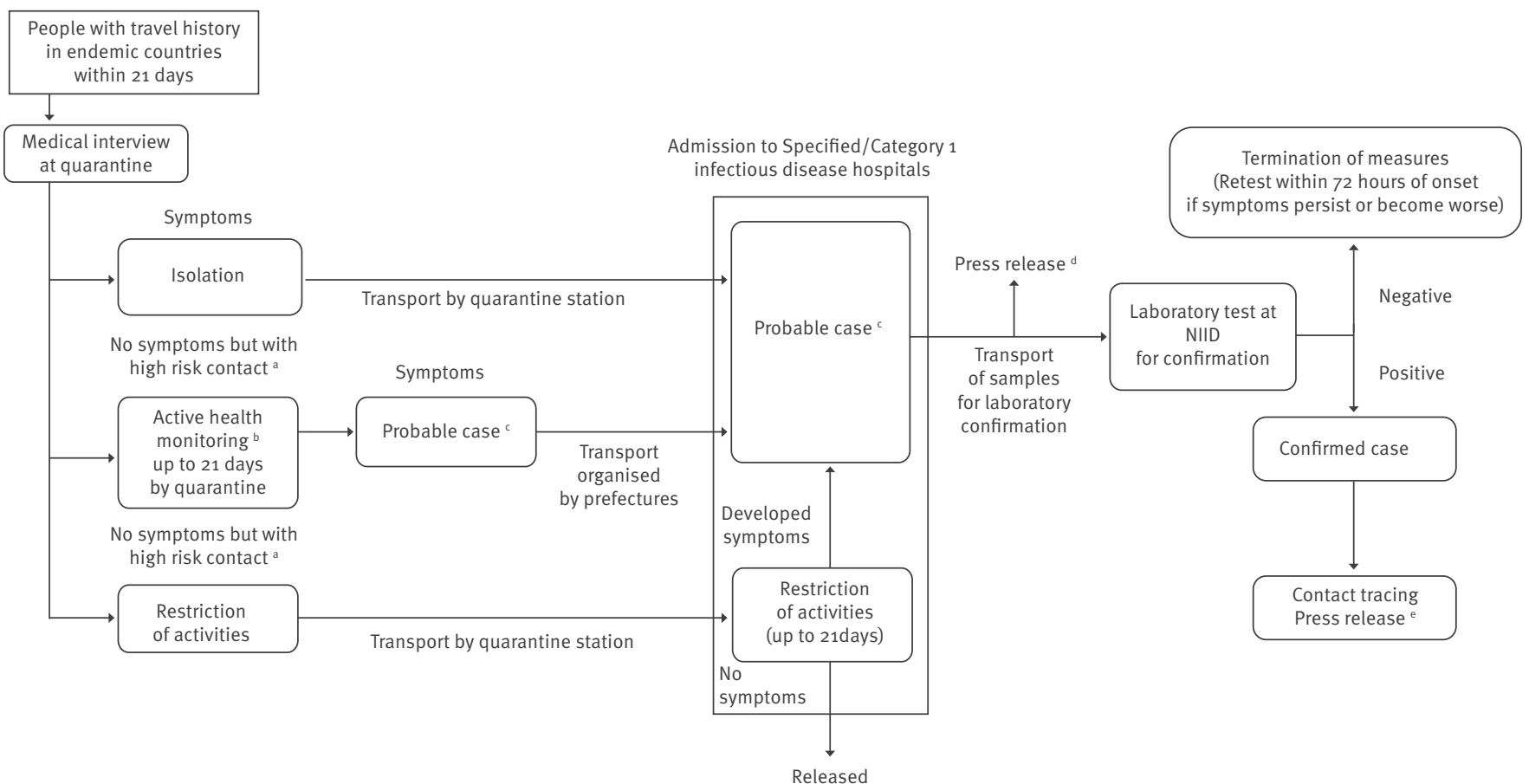

EVD: Ebola virus disease; NIID: National Institute for Infectious Diseases.

${ }^{a}$ High-risk contact i.e. direct contact with a virus through mucosa or wounds or by needle-stick injury .

b Individuals with at high risk of contact with a virus may be asked to stay at home.

'A probable case was defined on 24 October 2014 , as i) travel history to endemic countries within 21 days and fever OR ii) contact history to body fluids of patients and feeling of warmth. In order to emphasise the contact history, the definition was modified on 18 September 2015 as i) fever $>38^{\circ} \mathrm{C}$ or clinical symptoms of EVD AND 2) history of contact with body fluids through EVD patients OR history of contact with bats and primates in endemic countries within 21 days.

${ }^{d}$ A press release will be issued when a possible case is identified and blood samples are sent from the hospital to the NIID for diagnosis. The following patient data will be disclosed: age group, sex, prefecture of residence or nationality, travel history, contact history in the endemic country, symptoms, diagnosis of other infectious diseases, flight information for those developing symptoms at quarantine, prefecture of admitting hospital, day of departure from the endemic country and day of onset of symptoms developed during health monitoring [7].

${ }^{\text {e }}$ A press release will be issued when the case is laboratory-confirmed.

travellers entering Japan at quarantine stations from 1 August. Travellers were asked to declare their travel history to endemic countries and to contact local public health centres if they developed symptoms described in several communication channels, such as posters at quarantine stations and in-flight announcements. Starting with 24 October 2014 (at airport) and with 21 November (at seaport), immigration control officer also asked travellers if they had been in or travelled from an endemic country. Those with a history of contact with EVD patients were isolated if they had symptoms, or were placed under health monitoring [7].

The GOJ considered strengthening border measures after several reports of cases in non-endemic countries from September 2014 [2,3], and a large increase in numbers of patients in endemic countries. The case of a patient in Japan who had a history of travel to Liberia but did not attend a quarantine station and visited a hospital without his travel history being noted [8], further convinced the GOJ of the need to strengthen the management of people with a travel history to endemic countries.

On 21 October 2014, the MHLW revised the entry screening policy to define those who had travelled from Guinea, Liberia, or Sierra Leone within 21 days as having a history of contact with Ebola patients [7]. According to the Quarantine Act, these travellers were to be isolated if they had symptoms at quarantine, or to be put under active health monitoring i.e. having to report body temperature and conditions to a quarantine station twice a day for 21 days after the last visit to any of the three countries. The definition of a probable 
Function and roles of designated infectious disease hospitals under the Infectious Disease Control Act, Japan, as at 10 November 2014

\begin{tabular}{|c|c|c|c|}
\hline & \multicolumn{3}{|c|}{ Type of infectious disease hospital } \\
\hline & Specified & Class 1 & Class 2 \\
\hline Designated by & $\begin{array}{l}\text { Minister of Health, Labour and } \\
\text { Welfare }\end{array}$ & Prefectural Governor & Prefectural Governor \\
\hline Location policy & Several nationally & One in a prefecture & One in a secondary medical care area ${ }^{e}$ \\
\hline $\begin{array}{l}\text { Major requirements for } \\
\text { the wards }\end{array}$ & Not specifically documented & $\begin{array}{c}\text { Negative pressured private room with } \\
\text { toilet and shower } \\
\text { Anteroom } \\
\text { Dedicated ventilation with HEPA filter } \\
\text { Dedicated drainage }\end{array}$ & $\begin{array}{c}\text { Dedicated ward for infectious disease } \\
\text { patients with toilet and shower }\end{array}$ \\
\hline Diseases & $\begin{array}{c}\text { Novel }^{\mathrm{a}} \\
\text { Category }^{1^{\mathrm{b}}}, 2^{\mathrm{c}}\end{array}$ & Category $1^{\mathrm{b}}, 2^{\mathrm{c}}$ & Category $2^{\mathrm{C}}$ \\
\hline Hospitals (beds) & $3(8)^{d}$ & $\begin{array}{c}45(86)^{d} \\
\text { in } 38 \text { of } 47 \text { prefectures }\end{array}$ & $335(1,716)$ \\
\hline
\end{tabular}

HEPA: high efficiency particulate air.

a Novel infectious disease is a category for an emerging severe and highly transmissible disease with unknown aetiology

${ }^{b}$ Category 1 infectious diseases include smallpox, Ebola haemorrhagic fever, Marburg disease, Crimean-Congo haemorrhagic fever, Lassa fever, South American haemorrhagic fever and plague.

'Category 2 infectious diseases include acute poliomyelitis, diphtheria, severe acute respiratory syndrome, tuberculosis, and specified avian influenza virus infections ( $\mathrm{H}_{5} \mathrm{~N}_{1}$ and $\left.\mathrm{H}_{7} \mathrm{~N} 9\right)$.

d Two hospitals are designated as both Specified and Class 1 infectious disease hospitals.

e Approximately one in several municipalities

case which should be hospitalised in a designated hospital, as well as a confirmed case, was modified to emphasise the travel history to endemic countries (Figure). Thus, if people developed symptoms during monitoring, they were to self-isolate, call a quarantine station, and be hospitalised in a designated hospital. The response protocol for local health authorities was revised accordingly on 24 October 2014. The MHLW added measures on 21 November for those who were asymptomatic but had a high risk of EVD, asking them to stay home (Table 3) [9].

\section{Challenges in management of people travelling to Japan from endemic countries} Measures for Category 1 diseases under the Act and the Quarantine Act can be taken not only for a confirmed case, but also for a probable case, which is clinically diagnosed but not laboratory-confirmed. These measures were intended to be implemented as early as possible for those who had a high probability of infection when they developed symptoms. Because the early symptoms of VHFs are non-specific, the definition of a probable case should be applied cautiously to avoid imposed restriction of freedom of movement.

The modified definition of a probable case increased the sensitivity for detecting people at risk of EVD, and may have reassured the media and public when there was an epidemiological uncertainty about transmission mechanisms. There was a concern that those who visited endemic countries may have not recognised the contact to EVD patients. However, a higher sensitivity of a case definition may result in a larger number of people incorrectly classified as cases, for which unnecessary measures are enforced. A case reported on 7 November 2014 during active health monitoring had to be transported in an isolated unit from home to hospital under intense media attention, even though he did not have a contact history with EVD patients, and had been diagnosed with tonsillitis at a local clinic. Measures which are too strict and disproportionate may make people hesitate or reluctant to declare their symptoms voluntarily, which is the basis of modern policies of infectious disease control, and this will result in a negative impact on public health. In fact, the above mentioned case visited a clinic without giving notice to a quarantine station and did not tell the doctor about his travel history to Liberia despite instructions from a quarantine station during active health monitoring [10].

The protocol above had been active until it was revised on 18 September 2015, to emphasise the contact history to a patient or bats for the definition of a probable case. Special measures such as described here should be applied for a limited duration only and be reviewed periodically for appropriateness as the scenario changes to balance public health needs and rights of the individual of free movement.

The legacy in case management was a communication policy on possible EVD cases identified at quarantine and within the country (Figure) [7] to clarify the timing and contents of disclosure. The disclosure policy on a 
Cases screened for Ebola virus disease, Japan, August 2014-September 2015 (n=9)

\begin{tabular}{|c|c|c|c|c|c|c|c|}
\hline $\begin{array}{l}\text { Age groups } \\
\text { (years) }\end{array}$ & Sex & $\begin{array}{l}\text { Visited } \\
\text { country }\end{array}$ & Nationality & Symptoms & Contact history & Reporting & Diagnosis \\
\hline $\begin{array}{l}20-29(n=1) \\
30-39(n=2) \\
40-49(n=4) \\
50-59(n=0) \\
60-69(n=1) \\
70-79(n=1)\end{array}$ & $\begin{array}{c}\text { Male }(n=7) \\
\text { Female }(n=2)\end{array}$ & $\begin{array}{c}\text { Guinea }(n=4) \\
\text { Liberia }(n=3) \\
\text { Sierra } \\
\text { Leone }(n=2)\end{array}$ & $\begin{array}{c}\text { Japan }(n=6) \\
\text { Guinea }(n=2) \\
\text { Undisclosed }(n=1)\end{array}$ & $\begin{array}{c}\text { Fever }(n=9) \\
\text { Body pain }(n=2) \\
\text { Chill }(n=1) \\
\text { Cough }(n=1) \\
\text { Headache }(n=1)\end{array}$ & $\begin{array}{c}\text { None }(n=7) \\
\text { Contact to body } \\
\text { bag }(n=1) \\
\text { Undisclosed }(n=1)\end{array}$ & $\begin{array}{c}\text { During health } \\
\text { monitoring }(n=6) \\
\text { At } \\
\text { quarantine }(n=3)\end{array}$ & $\begin{array}{c}\text { Malaria }(n=4) \\
\text { Influenza }(n=1) \\
\text { Others }(n=4)\end{array}$ \\
\hline
\end{tabular}

\section{TABLE 3}

Classification of contact with confirmed cases of Ebola virus disease or related fluids [modified from reference 24], Japan, as at 21 November 2014

\begin{tabular}{|l|c|c|}
\hline Type of exposure & Appropriately protected & Unprotected or inappropriately protected \\
\hline $\begin{array}{l}\text { Direct contact with a virus to mucosa or wounds or by } \\
\text { needle-stick injury }\end{array}$ & NA & ${\text { Low risk }{ }^{\text {a }}}^{\text {High risk }{ }^{b}}$ \\
\hline Contact with body fluids of cases & Low risk $^{\text {a }}$ & High risk $^{\text {b }}$ \\
\hline Those handling specimens of cases & Low risk $^{\text {a }}$ & High risk $^{\text {b }}$ \\
\hline $\begin{array}{l}\text { Medical examination, procedure or transportation of cases } 1 \text { meter } \\
\text { Other staff involved in the medical management or } \\
\text { transportation of cases, those living with the cases }\end{array}$ & Low risk $^{\text {a }}$ & High risk $^{\text {b }}$ \\
\hline
\end{tabular}

NA: not applicable.

a Should be under active health monitoring without movement restriction.

b Should be under active health monitoring and are asked to stay at home.

case of an emerging disease has always been an issue of concern [11], and the protocol will provide a basis for such an event in the future.

\section{Collaboration of public health sectors with other agencies}

The GOJ activated the government-wide crisis response system on 28 October 2014 , immediately after the first traveller returning from Liberia to Japan developed fever at quarantine. The first ministerial meeting on the response to EVD was held with the participation of the Prime Minister. After that, the response and measures of relevant ministries and agencies were coordinated at the Intergovernmental Coordination Meeting on EVD measures, chaired by the Deputy Chief Cabinet Secretary for Crisis Management [12].

Progress was made in coordinating public health agencies with the fire department and police to safely transport patients and clinical samples. The Ministry of Internal Affairs and Communications agreed with the MHLW on the arrangements for transportation of confirmed or probable cases of EVD by the local fire departments when requested by local public health centres [13]. The National Police Agency also agreed with the MHLW on the assistance required for emergency transportation of a confirmed or probable case of Class 1 disease and its clinical samples [14]. These documented agreements represent a key legacy for future cooperation among the relevant agencies.

Planning and preparedness should be tested by regular simulation exercises to strengthen the collaboration between the relevant agencies. On 3 November 2014, the MHLW requested local governments to practice the protocol for transporting patients and samples. All 141 local governments and municipalities with public health centres completed the exercises before the end of March 2015. The next challenge is to maintain this collaborative network.

\section{Challenges for domestic medical response capacity for Category 1 diseases}

The primary strategy to provide medical care for VHF patients in Japan (population: 127 million as of May 2015 [15]) was to place a designated Class 1 hospital in each prefecture, because the authority for controlling infectious diseases is decentralised to 47 prefectures under the Act. Even though the incidence of Category 1 diseases may be very low, Class 1 hospitals are expected to play a role as core hospital to improve medical care for infectious diseases in the prefecture [16]. The cost of establishing and operating a Class 1 hospital has been subsidised directly by prefectures 
and indirectly by the MHLW; however, even 15 years after the Act was enforced, nine prefectures had not yet set up a Class 1 hospital at the declaration of the PHEIC in August 2014; two prefectures did however designate Class 1 hospitals in November 2014 and March 2015 [7]. To improve response capacity, training in the use of personal protective equipment was provided for 328 local public health officials and workshops were provided in 19 of 45 Class 1 hospitals from October 2014 to February 2015. The National Center for Global Health and Medicine, a Specified hospital, prepared a team to assist a Class 1 hospital in case of an emergency [12].

Maintaining high-level biosafety care facilities and trained staff in every prefecture may have been a too idealistic goal for continued capability in dealing with rare diseases. We have not experienced a Category 1 disease since 1999. Several people have been tested for VHF in the National Institute of Infectious Diseases of Japan every year, and all were negative [7]. Even under the raised screening sensitivity at quarantine in response to this large outbreak in West Africa, only nine cases were screened for EVD. A research group funded by the MHLW developed training programmes for staff in Class 1 hospitals, but only three-quarters of hospitals participated during 2011-13 [17]. In addition, the fact that only 29 of 45 Class 1 hospitals have infectious disease specialists qualified by the Japanese Association for Infectious Diseases, indicates that Class 1 hospitals have not played their expected role as core hospital for infectious diseases in the regions. Although any prefecture should be prepared for the appearance of highly infectious and pathogenic diseases with an appropriate level of biosafety, a more centralised strategy would more effectively concentrate available expertise and capacity for treatment of such extremely rare diseases in a sustainable manner.

The next important step would be to further improve practices in designated hospitals such as infection control measures in a biosafety ward. Specified hospitals should have a role in liaising with Class 1 hospitals to provide expertise, training, and simulation exercises. The legacy was the establishment of the Expert Committee for Treatment of Category 1 Diseases at the MHLW in October 2014. The Committee discussed therapeutic protocols, including the use of unapproved treatments. This platform will facilitate the sharing of up-to-date knowledge and of limited expertise for treatment and management of such rare diseases, and will support clinicians who care for patients with Category 1 diseases.

\section{Emergency use of medical countermeasures}

Favipiravir (Toyama Chemicals Co., Japan) is a drug licensed in Japan and indicated for infections with novel or re-emerging influenza viruses, although its use is limited to cases in which other anti-influenza virus drugs are ineffective or not sufficiently effective. Favipiravir is not yet marketed, and can only be distributed by order of the MHLW in the case of an emergency in an influenza pandemic. Although Favipiravir is not approved for EVD, it is expected that it can be used for EVD, as its efficacy for EVD has been shown in a mouse model [18]. There was a stock for 20,000 courses in tablet form, and for roughly 300,000 courses in active ingredients, in influenza treatment doses, in the company, as at 20 October 2014 [19].

Japan does not have an official access programme for use of unlicensed drugs outside clinical trials. The official view of the GOJ was that 'use of an unlicensed drug at a physician's discretion may be allowable' [20] and 'may not violate the Act on Pharmaceutical Affairs in this emergency situation' [21]. There remains a concern that there is no monitoring scheme for the efficacy and safety of unlicensed drugs, and no framework for an ethical consideration of their use. In response, the Expert Committee for Treatment of Category 1 Diseases recommended on 24 October 2014 that clinical data should be collected and shared with the public on the use of an unapproved drug [22]. A clinical research protocol for use of unapproved treatments in a Specified hospital was formulated. Such a framework prepared in advance should help provide the best emergency therapeutic options in an ethical manner, and track efficacy and safety of treatments for future emerging diseases without approved treatment options.

\section{Conclusions}

The EVD outbreak in West Africa provided a crucial opportunity to reveal challenges and improve preparedness for managing rare but high impact emerging diseases that are prone to be neglected. MHLW held a review meeting on the response to the EVD outbreak in West Africa to develop a technical guidance on preparedness and response to VHFs for public health agencies in October 2015 [23]. Some measures such as the case management policy may be country-specific; however, some challenges may not be specific to our country; for example, the strategy for sharing medical response capacity is a common concern for other countries, regions or at global level. Efforts to maintain achievements in public health preparedness as legacies may help contain future emerging diseases, including acts of bioterrorism and pandemic influenza.

\section{Acknowledgements}

I thank Professor Tsutomu Takeuchi (St. Luke's International University) for reviewing the manuscript and insightful comments. This research was supported by the Fund for Emerging and Re-emerging Infectious Diseases by the Japanese Agency for Medical Research and Development (15fk0108039ho02). This article contains the author's personal analysis and views, and does not represent the official position of the organisation.

\section{Conflict of interest}

None declared. 


\section{Authors' contributions}

Tomoya Saito performed the investigation and drafted the manuscript.

\section{References}

1. BriandS, BertheratE, CoxP, FormentyP, KienyMP, MyhreJK, et al. The international Ebola emergency. N Engl J Med. 2014;371(13):1180-3. DOI: 10.1056/NEJMp1409858 PMID: 25140855

2. Working group of Ebola outbreak investigation team of Madrid,LópazMA, AmelaC, OrdobasM, Domínguez-BerjonMF, ÁlvarezC, MartínezM, et al. . First secondary case of Ebola outside Africa: epidemiological characteristics and contact monitoring, Spain, September to November 2014. Euro Surveill. 2015;20(1):21003. DOI: 10.2807/1560-7917.ES2015.20.1.21003 PMID: 25613651

3. Centers for Disease Control and Prevention (CDC), ChevalierMS, ChungW, SmithJ, WeilLM, HughesSM, JoynerSN, et al. . Ebola virus disease cluster in the United States--Dallas County, Texas, 2014. MMWR Morb Mortal Wkly Rep. 2014;63(46):10878.PMID: 25412069

4. HirabayashiY, OkaS, GotoH, ShimadaK, KurataT, FisherHochSP, et al. An imported case of Lassa fever with late appearance of polyserositis. J Infect Dis. 1988;158(4):872-5. DOI: $10.1093 /$ infdis/158.4.872 PMID: 3171229

5. Paragraph 2, Article 6, Act on Prevention of Infectious Diseases and Medical Care for Patients of Infections. Act No.114 of 1998. [Accessed 3 November 2015]. Japanese. Available from: http:// law.e-gov.go.jp/htmldata/H10/H10H0114.html

6. Ministry of Justice. Immigration Control Statistics. [Accessed 3 November 2015]. Japanese. Available from: http://www.e-stat. go.jp

7. SaitoT, FukushimaK, AbeK, UjiieM, UmekiK, NakajimaK. Response to Ebola virus disease by the Ministry of Health, Labour and Welfare of Japan.Virus.2015;65(1):104-14.

8. OhshiroY, ShinzatoT. A falciparum malaria case who visited a community hospital after returning from West Africa.Infectious Agents Surveillance Report.2014;35:274-5.

9. Press Release from the Tuberculosis and Infectious Disease Control, Health Bureau, Office of Quarantine Station Administration, Policy Planning and Communication Division, Department of Food Safety, Pharmaceutical and Food Safety Bureau, Ministry of Health, Labour and Welfare. [Response to Ebola Hemorrhagic Fever at quarantine and in the country.]. 21 November 2014. Japanese. Available from: http://www.mhlw. go.jp/stf/houdou/0000066099.html

10. A man visited Liberia developed fever, Guinean lady in Kansa Airport, tested for Ebola. Nihon Keizai Shinbun. 8 November 2014 8. 13th edition; Sect. local news. Japanese.

11. SaitoT, FukushimaK, Umekik, NakajimaK. Severe fever with thrombocytopenia syndrome in Japan and public health communication.Emerg Infect Dis. 2015;21(3):487-9. DOI: 10.3201/eid2103.140831 PMID: 25695132

12. Intergovernmental Coordination Meeting on EVD measures. Response to Ebola Haemorrhagic Fever in Japan-Response so far and preparedness for the future -. Second Intergovernmental Coordination Meeting on EVD measures. 24 February 2015. Japanese. Available from: http://www.kantei. go.jp/jp/singi/ebola/

13. Notification the Tuberculosis and Infectious Disease Control Division, Health Bureau, Ministry of Health, Labour and Welfare. (No. 1128-1). [Collaboration of fire departments for a transport of a confirmed or probable case of Ebola Hemorrhagic Fever.] 28 November 2014. Japanese. Available from: http://www.mhlw.go.jp/bunya/kenkou/kekkakukansenshou19/dl/20141128_01.pdf

14. Notification from Community Police Affairs Division, Community Safety Bureau, National Police Agency (No. 171). [Cooperation on transport of clinical samples and patients related to Class1 Infectious Diseases (circular notice).] 30 October 2014. Japanese.

15. Official Statistics of Japan. Population Estimates by Age ( 5 Year Age Group) and Sex - May 1, 2015(Final estimates), October 1, 2015(Provisional estimates). Available from: http://www.e-stat. go.jp/SG1/estat/ListE.do?lid=000001138964

16. TakedaY, NomuraT. [Future Direction of Medical Care System for Patients with Infectious Diseases Control Law in Japan - centering around a category 1 hospital]. Japanese. Kansenshogaku Zasshi. 2000;74(9):687-93. DOI: 10.11150/ kansenshogakuzasshi1970.74.687 PMID: 11068360

17. Research group for clinical management and contact tracing of viral hemorrhagic fevers in Japan. Viral Hemorrhagic Fevers:
Guidance for Clinical Management. First edition. March 2014. Japanese.

18. OestereichL, LüdtkeA, WurrS, RiegerT, Muñoz-FontelaC, GüntherS. Successful treatment of advanced Ebola virus infection with T-705 (favipiravir) in a small animal model.Antiviral Res. 2014;105:17-21. DOI: 10.1016/j. antiviral.2014.02.014 PMID: 24583123

19. Government of Japan. Answers for Memorandum of Questions No. 33 of House of Councillors, the 187th National Diet of Japan. 28 October 2014. Japanese. Available from: http:// www.sangiin.go.jp/japanese/joho1/kousei/syuisyo/187/touh/ t187033.htm

20. Government of Japan. Answers for Memorandum of Questions No. 29 of House of Representatives, the 187th National Diet of Japan. 24 October 2014. Japanese. Available from: http://www. shugiin.go.jp/internet/itdb_shitsumon.nsf/html/shitsumon/ a187029.htm

21. Summary of post-cabinet meeting press conference by Minister of Health, Labour and Welfare. 15 August 2014. Available from: http://www.mhlw.go.jp/stf/kaiken/daijin/o000054819.html. Japanese.

22. Summary of the Expert Committee for treatment of Category 1 Infectious Diseases. 24 October 2013.http://www.mhlw.go.jp/ stf/shingi2/0000063142.html

23. Tuberculosis and Infectious Disease Control Division, Health Bureau, Ministry of Health, Labour and Welfare. The first review meeting on Class I infectious diseases. 20 October 2015. Japanese. Available from: http://www.mhlw.go.jp/stf/ shingi2/0000101839.html

24. National Institute for Infectious Diseases. Interim Manual of active epidemiological investigation for EVD. November 21 , 2014. Japanese. Available from: http://www.nih.go.jp/niid/ images/epi/ebola/1121.pd 